\title{
Patterns of sweetness preference in red wine according to consumer characterisation
}

\author{
Maria Madalena Sena-Esteves, Mariana Mota, Manuel Malfeito-Ferreira* \\ Linking Landscape, Environment, Agriculture and Food Research Center (LEAF), Instituto Superior de Agronomia (ISA), University of Lisbon (UL), Tapada da Ajuda, \\ 1349-017 Lisboa, Portugal
}

\section{A R T I C L E I N F O}

\section{Keywords:}

Consumer categories

red wine

Sweetness preference

2-AFC method

Personality traits

\begin{abstract}
A B S T R A C T
The preference for sweet taste in red wine was examined according to consumer categories of age, gender, drinking experience and personality type (Big-5 personality-test). A total of 114 subjects revealed their preferences for sweetness after tasting dry red wine spiked with equal concentrations of glucose and fructose at $2 \mathrm{~g} /$ $\mathrm{L}, 4 \mathrm{~g} / \mathrm{L}, 8 \mathrm{~g} / \mathrm{L}, 16 \mathrm{~g} / \mathrm{L}$ and $32 \mathrm{~g} / \mathrm{L}$, following an ascending forced choice paired comparison method (2-AFC).

The overall preference for sweetness was shown within the range of 4.8 to $21.9 \mathrm{~g} / \mathrm{L}$, with maximal liking at $8 \mathrm{~g} / \mathrm{L}$. Three patterns of response to sweetness were observed (sweet dislikers, sweet likers and indifferent to sweet) according to the different categories of consumers. Differences $(p>0.05)$ were not found in sweetness preference among the categories up to $16 \mathrm{~g} / \mathrm{L}$ sugar except for the trait extraversion at $8 \mathrm{~g} / \mathrm{L}$, where low extraverts showed a higher proportion of responses preferring the sweeter sample. Most significant differences were found only under the highest tasted concentration $(32 \mathrm{~g} / \mathrm{L})$. Females and novices preferred sweeter samples $(p<0.05)$ when compared with the response of males and experienced consumers, respectively.
\end{abstract}

\section{Introduction}

The sensory properties of a wine are a major element that will determine its success among consumers (Francis \& Williamson, 2015). These authors provided an overview of the current knowledge of the sensory attributes that have been found to be important to consumer preference and liking. The relationships between consumer hedonic response and wine sensory attributes, as quantified by a trained sensory panel, help enable the understanding of the sensory characteristics that drive acceptance. A common finding is the importance for many consumers of the appropriate balance among acidity (sourness) (Francis et al., 2010; Lésschaeve, Bowen, \& Bruwer, 2012), bitterness and sweetness (Francis et al., 2010), and astringency (Lattey, Bramley, \& Francis, 2010). These mouthfeel properties are complemented by aromatic features, where fruity, floral and oak flavours are particularly appreciated (Lund et al., 2007, Marchal, Pons, Lavigne, \& Dubourdieu, 2013). Comparatively, studies concerning preferences for sweet mouthfeel are fewer and present knowledge is mostly based on early research (Amerine \& Ough, 1967; Duitschaever, Buteau, \& Ashton, 1980; Filipello, Berg, Hinreiner, \& Webb, 1955; Kielhöfer, 1955). More recently, Blackman, Saliba, and Schmidtke (2010) reported the preference for sweetness in two Australian Semillon wines with different acidity levels. International wine challenges also tend rate sweeter samples more highly (Loureiro, Brasil, \& Malfeito-Ferreira, 2016). Therefore, either from controlled experiments with wines spiked with different sugar levels or from overall consumer preferences, it seems that a "sweet tooth" observed in other foods (Wansink, Bascoul, \& Che, 2006) may also be applied for wine drinkers.

Preference for sweet foods has been studied associated with the perception of sweetness intensity revealing several patterns of sweet liking (Drewnowski, Henderson, Shore, \& Barratt-Fornell, 1997; Kim, Prescott, \& Kim, 2014; Thompson, Moskovitz, \& Campbell, 1976 and Whiterly, Pangborn, \& Stern, 1980). Intensity ratings of sweetness show that consumers with different sweet liking status have similar intensity ratings either in water (Drewnowski et al., 1997; Kim et al., 2014) or in foods and beverages (Drewnowski et al., 1997; Kim et al., 2014; Methven, Xiao, Cai, \& Prescott, 2016). Therefore, sweet perception appears to be independent from the pleasantness of sweetened foods, although a recent report stated otherwise (Jayasinghe et al., 2017). The former authors established sugar preference or acceptance dose-response curves with several shapes, yielding 3 or 4 types of hedonic responses, which may be reduced to 2 , gathering sweet/sucrose likers in opposition to sweet/sucrose dislikers (Drewnowski et al., 1997, Methven et al., 2016, Yeomans, Prescott, \& Gould, 2009, Yeomans, Tepper, Riestzschel, \& Prescott, 2007). The establishment of individual sweet liking status may be obtained across multiple sugar

\footnotetext{
* Corresponding author.

E-mail address: mmalfeito@isa.ulisboa.pt (M. Malfeito-Ferreira).
} 
concentrations using hedonic Likert scales (Drewnowski et al., 1997), visual analogue scales (Kim et al., 2014; Methven et al., 2016; Yeomans et al., 2007), labeled magnitude scales (Jayasinghe et al., 2017; Yeomans et al., 2007) or paired comparison tests (Blackman et al., 2010). Yeomans et al. (2009) used two concentrations of sucrose and two concentrations of saccharin to classify sweet liking. Hedonic responses to one concentration of sugar (Kim et al., 2014; Saliba, Wragg, \& Richardson, 2009) failed to reveal some features of liking patterns when compared to ratings across increasing concentrations (Kim et al., 2014). However, for rapid screening tests a single concentration may be used to separate between sweet likers and sweet dislikers (Asao et al., 2015).

Consumer segmentation is regarded as essential to understand preferences for different types of wine (Francis \& Williamson, 2015). In other food and beverage products, it has been shown that the response to sweet taste by consumers is highly segmented (Kim et al., 2014; Moskovitz, Jacobs, \& Lazar, 1985). In wines most frequent studied segments or categories include gender, age, level of expertise, consumption habits or culture (Blackman et al., 2010; Lattey et al., 2010; Lésschaeve et al., 2012; Osidacz, Francis, Bramley, \& Stevens, 2011; Williamson, Robichaud, \& Francis, 2012). Personality traits have also been shown as influencing food choice. The research on this theme relies on the 'Big Five' theory of personality that involves the independent traits of extraversion, neuroticism, openness, conscientiousness and agreeableness (Hogan, Johnson, \& Briggs, 1997). Extraversion reflects how much the individual is oriented towards things outside him and derives satisfaction from interacting with other people. Neuroticism is the tendency to experience negative emotions. Openness to experience has been portrayed as a proxy measure of the willingness to explore new and unfamiliar experiences, ideas and feelings, while conscientiousness refers to persistence, perfectionism and self-discipline. Agreeableness means being sympathetic, kind, affectionate and reflects how much an individual likes and tries to please others (Costa Jr. \& McCrae, 1992). A preference for sweet and salty tastes in people who score high in the neuroticism trait was found by Kikuchi and Watanabe (2000). In wines, Saliba et al. (2009) found that sweet taste preference was associated with a higher level of impulsiveness but lower openness. A study involving aroma characterization and liking of a set of Italian red wines showed a link between "innovativeness" (related to openness) and consumers who could reliably differentiate samples (Torri et al., 2013).

Wine is a complex matrix with several taste and mouthfeel interactions. In white wine sugar reduces sourness while in reds reduce astringent and bitter sensations (Sáenz-Navajas, Fernandez-Zurbano, \& Ferreira, 2012). Therefore, the addition of sugar may result in lowering the perception of these attributes while sweetness remains unnoticeable. According to our empirical experience, the appeal of smooth red wines has led winemakers to increase residual sugar to relatively high levels, far beyond the level of balancing excessive astringency or bitterness. Therefore, it seems relevant to provide wine industry with guidelines on the addition of sugar to increase red wine acceptance in parallel to what has been reported for white wines (Blackman et al., 2010). The research described in this work was based on Blackman et al. (2010) using a larger number of consumers segmented in several traits other than individual expertise. The main objective was to establish a relationship between the preference for sweetness in a red wine and the different consumer age, gender, wine drinking experience and personality type, hoping to help winemakers in tuning up the levels of residual sugar in red wines according to each consumer target.

\section{Material and methods}

\subsection{Consumer categories}

One hundred and fourteen participants were recruited from ISA (Instituto Superior de Agronomia, Lisbon, Portugal) and other external
Table 1

Number of subjects in each class of the categories under study.

\begin{tabular}{lll}
\hline Categories & Classes & Number of subjects \\
\hline Age & {$[18-24]$} & 68 \\
& {$[25-29]$} & 27 \\
Gender & {$[30-60]$} & 19 \\
& Females & 51 \\
Experience & Males & 63 \\
Agreeableness & Novices & 85 \\
& Experienced & 29 \\
Neuroticism & A high & 113 \\
\multirow{2}{*}{ Extraversion } & B low & 1 \\
\multirow{2}{*}{ Openness } & A high & 45 \\
& B low & 69 \\
Conscientiousness & A high & 88 \\
& B low & 26 \\
& A high & 100 \\
& B low & 14 \\
& A high & 94 \\
\hline
\end{tabular}

faculties such as medicine, law and engineering. The average age of the participants was 27, ranging from 19 to 56 years old. Consumers were initially asked to answer some questions in order to group them into several classes in the following categories: gender, age, wine drinking experience and personality type (Table 1 ).

The wine drinking experience included novice and experienced consumers, based on a combination of self-reported consumption, years of experience in drinking wine, wine knowledge, and involvement in the wine industry, consistent with previous research (Blackman et al., 2010; Melcher \& Schooler, 1996). Specifically, the definitions provided by Blackman et al. (2010) were followed: i) a novice was an individual who had been a wine consumer for $<10$ years, that drank less than three times a week and who did not self-report as being "knowledgeable"; ii) an experienced consumer was an individual who had been a wine consumer for $>10$ years, drinking at least several times a week and who self-reported as being "interested" or "knowledgeable"; iii) the respondents who reported being wine consumers for $<10$ years and "knowledgeable" were also classified as experienced consumers.

To evaluate personality, subjects took the Big 5 personality-test (Hogan et al., 1997), which consists in 50 questions to be answered online (www.personality-testing.info/tests/IPIP-BFFM/) on the 5 personality traits: openness to experience, conscientiousness, extraversion, neuroticism and agreeableness. These traits were rated from 1 to 5 , and arbitrarily grouped in two classes, high scorers (class A) for the scores from 3 to 5 and low scorers (class B) from 1 to 2, to obtain higher number of respondents in each class.

\subsection{Wines}

The wine was a 2014 blend of Syrah (80\%), Touriga Nacional, Cabernet Sauvignon and Trincadeira varieties (20\%) produced in the experimental winery of ISA following classical vinification with skin maceration until sugar dryness. The concentrations of residual sugar in wine were $2,4,8,16$ and $32 \mathrm{~g} / \mathrm{L}$, obtained by the addition of equal amounts of glucose and fructose (Sigma-Aldrich, St. Louis, USA). Wines were analyzed on $\mathrm{pH}$, total acidity, volatile acidity, free and total sulfur dioxide, reducing substances and alcohol strength using standard OIV (International Office of Vine and Wine) methods (OIV, 2010). The characterization of colour and phenolic compounds was performed by evaluating (i) colour intensity, tonality, total pigments, polymeric pigments, pigment polymerization index, total and stained anthocyanins (Somers \& Evans, 1977); (ii) tannic power (NTU/mL) (Freitas \& Mateus, 2001) and (iii) total phenols (mg/L of gallic acid) (Ribéreau-Gayon, 1970). 


\subsection{Testing procedure}

The tasting session took place in a well ventilated classroom from 11 am to 5 pm, in 4 different sessions held in December 2015. In each bench, other than the glasses of wine, there was a glass of water, a spittoon, the demographic questionnaire and a laptop for the respondents to take the "Big 5 Personality" test. First, they were required to complete the forms by replying to the questions with the first answers that came into their mind. Once the questionnaires had been completed, wine tasting was initiated.

To assess tasters response to sweetness, a two ascending forced choice (2-AFC) protocol was conducted following the procedures described by the ISO guidelines for two-tailed paired comparisons (ISO, 1983), accepting a 5\% risk of error, as applied by Prescott, Norris, Kunst, and Kim (2005) and Blackman et al. (2010).Participants were asked to compare the base wine (control) with the spiked wines at each sugar level and indicate their preference on a scoresheet. Base wine position (left or right) was randomized. No information was given on the wine such as its variety, origin, or nature of the test. Each paired sample had a control wine and a spiked wine which was presented in increasing sugar concentration. Respondents were instructed to taste the wine, expectorate, rinse their mouth with water and wait $1 \mathrm{~min}$ before going on to the next sample. The wine $(30 \mathrm{~mL})$ was poured at $18^{\circ} \mathrm{C}$ in transparent ISO standard glasses (Ideal 6019, Schott Zwiesel, Germany), labeled with randomized 3-digit numbers. Participants were also asked to write what type of mouth-feel difference they felt in the preferred sample. Time was given as much as they needed to complete the test and released after tasting all the samples.

\subsection{Data analysis}

The assessment of differences between each class within a consumer category was performed counting as 1 when the spiked sample was preferred and as 0 when the control wine was preferred. Pearson's Chisquare test was used to assess if there was any influence of the different categories on the sugar preference. Tests were performed with software $\mathrm{R}$ (www.r-project.org) for each sugar concentration. When a category significant influence $(p<0.05)$ was identified, post-hoc tests were conducted to evaluate the influence of each class within the category.

\section{Results}

\subsection{Consumer characterization}

The characterization of each consumer category is shown in Table 1. The minimum number of 40 respondents for each category class as recommended for consumer preference tests by MacFie (2007) was not always achieved, even after pooling some of the classes. However, the ISO-5495 standard tables present a minimum of 7 replies to establish significance with a $5 \%$ risk of error $(\alpha<0.05)$ applicable when tasters may be considered as experienced. Therefore, results were also statistically compared for the experienced segment with 29 participants.

\subsection{Wine chemical analyses}

The young red wine used in tastings was selected due to its noticeable astringency hoping that the added sugar could effectively increase preference. Although it was not expected that sugar could modify the polyphenolic composition, the measurements of colour and astringency indexes were performed to check if any change could occur. The results (Supplementary data 1) showed that there were no significant differences between the control and all the spiked samples $(p>0.05)$. In this way taster responses could be ascribed to the sole effect of sugar addition and not to other effect on other mouthfeel characteristics. Higher sugar concentrations $(64 \mathrm{~g} / \mathrm{L})$ did increase wine volume inducing a dilution effect in phenolic indexes and were not used.

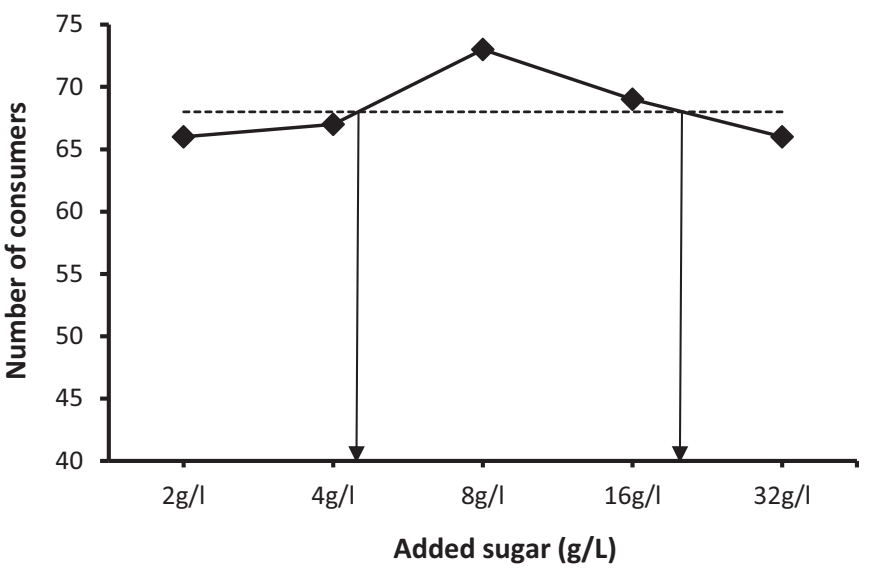

Fig. 1. Number of consumers $(\downarrow)$ who preferred the sugar spiked sample for each concentration. Dotted line $(n=68)$ represents minimum agreeing judgements necessary to establish preference using $\alpha=0,05$ for paired comparison tests when total the number of tasters was 114 . Vertical arrows indicate threshold values obtained by interpolation.

\subsection{Patterns of hedonic responses to sweetness according to consumer categories}

The overall response for increasing levels of sweetness is represented in Fig. 1 which shows the number of subjects who preferred the glucose and fructose spiked sample at each concentration. Using the 0.05 significance level (ISO, 1983) and interpolation between two neighboring concentrations, the spiked wine samples preferred to the control wine were those between $4.8 \mathrm{~g} / \mathrm{L}$ and $21.9 \mathrm{~g} / \mathrm{L}$, with a maximum of individuals preferring $8 \mathrm{~g} / \mathrm{L}$. The response curve describing the number of respondents preferring the spiked sample can be described as having an inverted-U shape. This overall behavior may be understood as the sum of the different responses given by each consumer segment. The response curve for each particular segment is described below.

The segment age was divided in 3 classes. Class A (18 to 24 years) preferred concentrations between 7.2 and $13.2 \mathrm{~g} / \mathrm{L}$, and above $25.6 \mathrm{~g} / \mathrm{L}$ up to $32 \mathrm{~g} / \mathrm{L}$ (Fig. 2). Classes B ( 25 to 29 years) and C (30 to 60 years) did not reach the threshold level to show preference for added sugar. However, class B showed decreasing proportion of tasters preferring higher sugar levels while Class $\mathrm{C}$ showed constant responses along the tested concentrations.

In the case of females, there was an increasing proportion of tasters

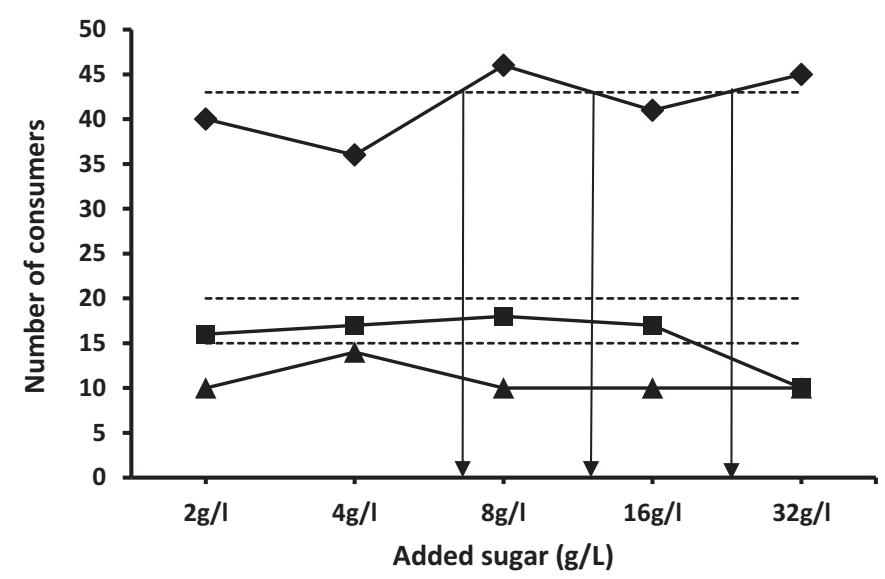

Fig. 2. Number of consumers with 18 to 24 years $(\diamond), 25$ to 29 years $(\square)$ and 30 to 60 years $(\boldsymbol{A})$ who preferred the sugar spiked sample for each concentration. Dotted lines ( $n=43,20$ and 15) represent minimum agreeing judgements necessary to establish preference using $\alpha=0,05$ for paired comparison tests when total the number of tasters were 68, 27 and 19, respectively. Vertical arrows indicate threshold values obtained by interpolation. 


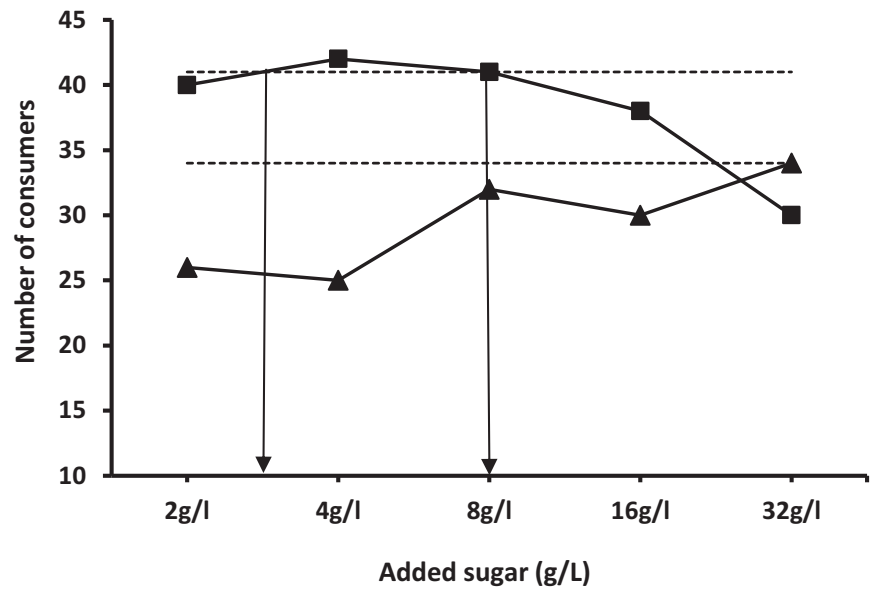

Fig. 3. Number of female $(\boldsymbol{\Delta})$ and male $(\boldsymbol{\square})$ consumers who preferred the sugar spiked sample for each concentration. Dotted lines $(n=34$ and 41$)$ represent minimum agreeing judgements necessary to establish preference using $\alpha=0,05$ for paired comparison tests when total the number of tasters were 51 and 63, respectively. Vertical arrows indicate threshold values obtained by interpolation.

preferring the spiked samples up to $32 \mathrm{~g} / \mathrm{L}$, concentration under which preference could be established for the sweeter wine (Fig. 3). In the case of males, the preference for sugar was observed between 3 and $8 \mathrm{~g} / \mathrm{L}$, with an inverted U-shape response curve.

The responses of the two classes of wine drinking experience are depicted in Fig. 4. The experienced consumers did not show a preference for sugar while the novice group showed preference over $7 \mathrm{~g} / \mathrm{L}$ up to the maximum level of $32 \mathrm{~g} / \mathrm{L}$.

The personality tests consider 4 possible scores for each trait: high A (4-5), medium M (3-4), low B (2-3) and very low MB (1-2). However, the few number of respondents in many of the categories led to their merging in two classes: high (A), for from 3 to 5, and low (B), for low from 1 to 2 . Even after these rearrangements, only one subject resulted low in agreeableness. Therefore, the high score in agreeableness tasters showed an inverted-U response similar to that of all subjects presented in Fig. 1 (results not shown). The trait neuroticism showed different behaviors for higher and lower scorers (Fig. 5a). Class A revealed an increasing proportion of tasters preferring the sweeter sample, showing preference over $26.7 \mathrm{~g} / \mathrm{L}$ sugar. Lower scorers, class B, revealed a response curve with an inverted $U$ shape, showing a significant preference

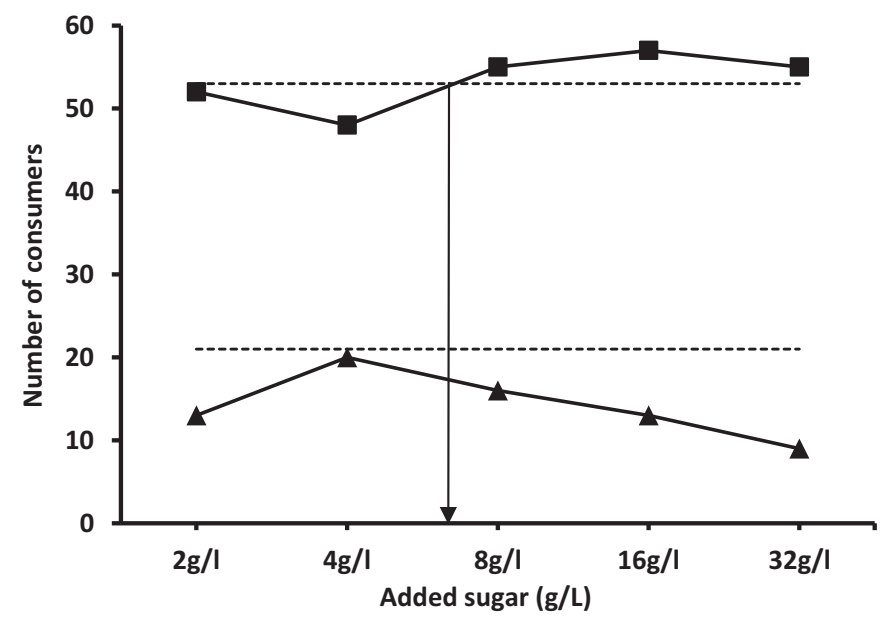

Fig. 4. Number of novices $(\boldsymbol{\square})$ and experienced $(\boldsymbol{\Delta})$ consumers who preferred the sugar spiked sample for each concentration. Dotted lines $(n=53$ and 21) represent minimum agreeing judgements necessary to establish preference using $\alpha=0,05$ for paired comparison tests when total the number of tasters were 85 and 29, respectively. Vertical arrows indicate threshold values obtained by interpolation. between 5.5 and $12.5 \mathrm{~g} / \mathrm{L}$. Regarding extraversion, the low scorers, class B, preferred concentrations between $3.6 \mathrm{~g} / \mathrm{L}$ and $14.1 \mathrm{~g} / \mathrm{L}$ (Fig. 5b). The higher scorers, class A, did not show preference as a function of sugar level. Concerning openness, the high scorers preferred the spiked sample with concentrations between 6 and $20.4 \mathrm{~g} / \mathrm{L}$ (Fig. 5c). Low scorers did not show preference for sugar at any of the tested concentrations. About conscientiousness, the high scorers preferred the spiked samples with concentrations between 4 and $24 \mathrm{~g} / \mathrm{L}$ (Fig. 5d). For the low scorers, preference for sweetness was not evidenced, being indifferent to the increase in sugar concentrations.

The several hedonic patterns of sugar liking reported by Thompson et al. (1976), Whiterly et al. (1980), Drewnowski et al. (1997) and Kim et al. (2014) were obtained with hedonic ratings across several sugar concentrations in the approximate range of $20 \mathrm{~g} / \mathrm{L}$ to $360 \mathrm{~g} / \mathrm{L}$. In the present study, sugar levels were about 10 -fold lower which, in preliminary tests, did not induce different liking scores using a visual analogue scale (results not shown). Therefore, preference was established through a 2-AFC protocol, as described by Blackman et al. (2010). Preference could only be demonstrated when number of responses choosing the sweetened sample were over a minimum value. Therefore, the curves presented in the figures reflect the overall response of the tasters and only show preference over the dotted line. These response curves have different shapes that can be grouped into 3 types (Table 2): (i) type I, defined as sweet dislike category, showing a decreasing or inverted-U shape proportion of tasters preferring the sweeter sample; (ii) type II, the sweet like category, defined as increasing proportion of individuals preferring sweeter samples and (iii) type III, indifferent to sweetness, defined as the responses without variation over the sugar concentrations. Table 2 shows that Type I (sweet dislikers) was the category that combined more segments: ages ranging from 25 to 29 years, males, experienced consumers, subjects who scored high on agreeableness, openness and conscientiousness and low on neuroticism and extraversion. Type II (sweet likers) was the category that included ages ranging from 18 to 24 years, females, novices and subjects who scored high on neuroticism. Type III (sweet insensitives), was the category that included subjects with 30 to 60 years old, scored high on extraversion, low on conscientiousness and low on openness. In addition, the utilization of a 2-AFC sensory technique provided an additional feature to the hedonic pattern related with the establishment of preference for sweetness. Type I and Type II included segments where preference for sugar was not demonstrated while all segments in Type III did not show preference for sweetness (Table 2).

\subsection{Comparison of sweetness preference among taster categories}

To compare the responses of each category, the output of each sugar concentration was considered as 1 , when the spiked sample was preferred, or 0 , when it was the case for the control sample. For each sugar concentration and each category, positive and negative responses were counted, and the proportion of positive answers calculated. The use of the Chi-square test for each sugar concentration showed that, most of the categories did not show significant different influence on sweetness preference (results not shown). For the trait extraversion, differences were found only at $8 \mathrm{~g} / \mathrm{L}(p=0.02)$, with low extraverts showing a higher preference $(0.85 \pm 0.07$, mean \pm standard error $)$ than high extraverts $(0.58 \pm 0.05$, mean \pm standard error). Significant differences were mainly found at the highest concentration tested $(32 \mathrm{~g} / \mathrm{L})$ for gender $(p=0.02)$ and experience $(p=0.01)$ categories. Females and novices showed higher proportion preferring the sweeter samples (Table 3).

The taster responses also suggested the existence of interactions within gender and category of wine drinking experience (at $8 \mathrm{~g} / \mathrm{L}$ sugar), and within neuroticism and openness (at $16 \mathrm{~g} / \mathrm{L}$ sugar). However, the comparison between the responses between classes within each category was not statistically valid taking in account the 

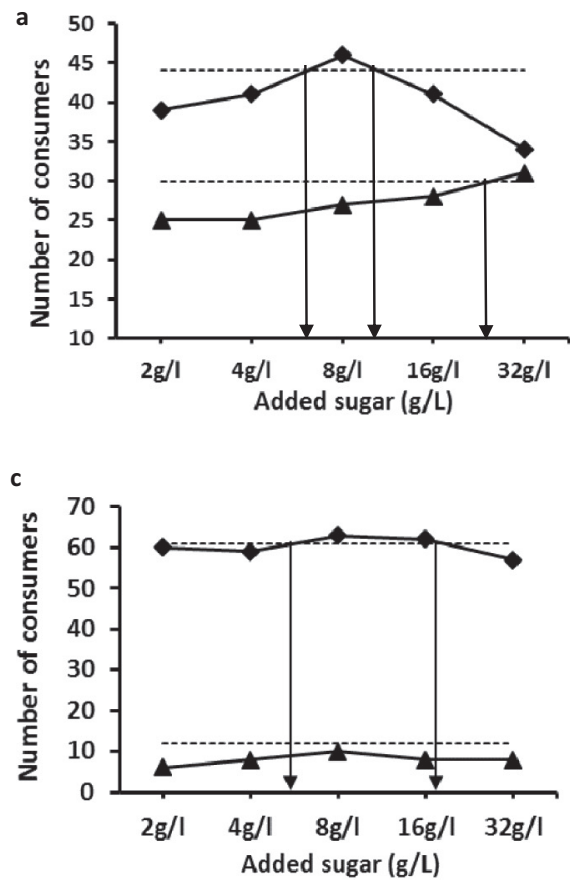
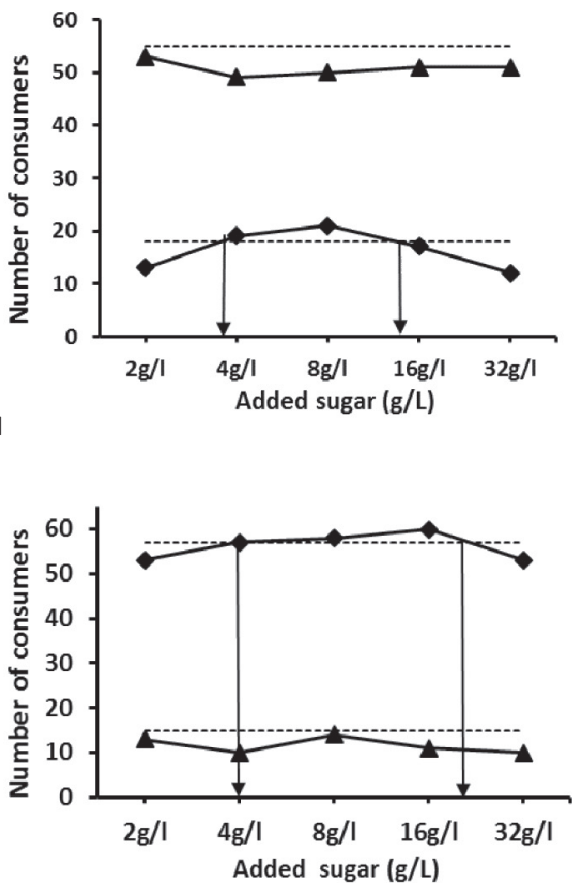

Fig. 5. Number of consumers with high $(\boldsymbol{A})$ and low ( $\bullet$ psychological traits who preferred the sugar spiked sample for each concentration: (a) Neuroticism, dotted lines $(n=30$ and 44) represent minimum agreeing judgements necessary to establish preference using $\alpha=0,05$ for paired comparison tests when total the number of tasters were 45 and 69, respectively; (b) Extraversion, dotted lines ( $n=18$ and 55) represent minimum agreeing judgements necessary to establish preference using $\alpha=0,05$ for paired comparison tests when total the number of tasters were 26 and 88, respectively; (c) Openness, dotted lines ( $n=61$ and 12) represent minimum agreeing judgements necessary to establish preference using $\alpha=0,05$ for paired comparison tests when total the number of tasters were 100 and 14, respectively; (d) Conscientiousness, dotted lines ( $n=58$ and 15) represent minimum agreeing judgements necessary to establish preference using $\alpha=0,05$ for paired comparison tests when total the number of tasters were 94 and 20 , respectively. Vertical arrows indicate threshold values obtained by interpolation.
Table 2

Consumer segments grouped according to the types of hedonic responses to sweetness. Type I - Inverted-U shape, type II - positive hedonic rating and type III - insensitive responses.

\begin{tabular}{llll}
\hline Type & Denomination & Consumer segments & $\begin{array}{l}\text { Preferred sugar } \\
\text { concentrations }(\mathrm{g} / \mathrm{L})\end{array}$ \\
\hline I & Sweet dislikers & Age (25-29) & No preference \\
& & Males & $3-8$ \\
& & Experienced & No preference \\
& & High openness & $6-20.4$ \\
& & High agreeableness & $4.8-21.9$ \\
& & Low neuroticism & $6.4-11.1$ \\
& & High conscientiousness & $4-24$ \\
II & Sweet likers & Low extraversion & $3.6-14.1$ \\
& & Age (18-24) & $2-8,24-32$ \\
& & Females & 32 \\
& & Novices & $7-32$ \\
III & Insensitive to & High neuroticism & 26.7 \\
& sweet & Age (30-60) & No preference \\
& & Low openness & No preference \\
& & Low conscientiousness & No preference \\
& & High extraversion & No preference \\
\hline
\end{tabular}

Table 3

Proportion of the tasters preferring the wine spiked with $32 \mathrm{~g} / \mathrm{L}$ sugar according to the different consumer categories that showed significant differences using a Chi-square test. Proportions of female and novices were significantly higher that proportions of male and experienced tasters $(\alpha=0.05)$.

\begin{tabular}{lllc}
\hline Gender & Mean & Experience & Mean \\
\hline Female & 0.71 & Novices & 0.65 \\
Male & 0.48 & Experienced & 0.36 \\
\hline
\end{tabular}

required minimum number of 40 respondents advised by MacFie (2007) for each consumer class (results not shown).

\section{Discussion}

The results from this work showed that dry red wine preference is clearly influenced by the residual sugar concentration. When responses from all individuals were pooled together, red wines with sugar between 4.8 and $21.9 \mathrm{~g} / \mathrm{L}$ were preferred over the control wine. The strongest preference was observed for the wine that had been spiked with $8 \mathrm{~g} / \mathrm{L}$ sugar, following an inverted U-shape pattern. This behavior, defined as sweet disliking, is common in foods where patterns of individual hedonic response to increasing levels of sweetness have been reported (Drewnowski et al., 1997; Kim et al., 2014; Methven et al., 2016; Moskovitz et al., 1985; Prescott, 1998; Prescott et al., 1992; Thompson et al., 1976; Whiterly et al., 1980; Yeomans et al., 2007). In the present work, probably due to the low sugar concentrations tested, hedonic scales did not show differences in liking. Therefore, the method of choice was a 2-AFC technique that enabled to observe a pattern across multiple sugar concentrations, as advised by Kim et al. (2014) and also validated by Blackman et al. (2010), in white wines, Harwood, Ziegler, and Hayes (2012), in chocolate milk, and Methven et al. (2016), in orange juices or jellies. The utilization of higher sugar concentrations would have made no sense because wines could no longer be regarded as dry. In fact, the higher sugar $(64 \mathrm{~g} / \mathrm{L})$ level would fall in the sweet wine category (OIV, 2015), besides that the required spiked solution would dilute wine polyphenols with effects in wine preference that could not be only ascribed to sugar addition (see Table 2 in supplementary material). This inverted-U shape behavior is reported for the first time regarding sugar level in red wines. In white wines, decreasing preference with (for novices and experienced consumers) or without an initial plateau (for winemakers) was described by Blackman et al. (2010). These authors reported maximum preference thresholds ranging from $2 \mathrm{~g} / \mathrm{L}$ glucose for winemakers to $16 \mathrm{~g} / \mathrm{L}$ for novices, both consistent with a sweet disliking pattern but without an inverted-U shape. Following the same 2-AFC technique, qualitatively similar results were found in certain consumer segments but the decrease in preference with higher sugar concentration was more pronounced in white wines tasted by winemakers and experienced individuals. Moreover, the sweet liking and insensitive to sweetness patterns were not reported by Blackman et al. (2010). These differences might be due to the astringency/bitterness of red wines. Knowing that sweetness masks bitterness and the puckering effect of astringency (Sáenz-Navajas et al., 2012), the presented results reflect the same behavior reported by 
Lattey et al. (2010) where the observed inverted-U shape curve for liking of different red wines was attributed to varying levels of astringency. Accordingly, similar inverted-U shape curves with optimum sucrose levels at $18 \mathrm{~g} / \mathrm{L}$ were observed in tea, a non-alcoholic bitter beverage (Chung \& Vickers, 2007).

The preference for wine with added sugar can also be inferred from other reports where the objective was to assess liking of different wines. Lattey et al. (2010), in a range of Shiraz and Cabernet Sauvignon wines, showed that the sweetest wine $(7.1 \mathrm{~g} / \mathrm{L})$ was the most preferred by Australian consumers and received intermediate scores by winemakers. Williamson et al. (2012), studying Chinese and Australian consumers' liking responses for red wine, showed that they share broadly similar preferences, preferring the wine with $16.1 \mathrm{~g} / \mathrm{L}$ sugar. In Austria, with white wines, the most appreciated wine had $10.8 \mathrm{~g} / \mathrm{L}$ sugar although tasters did not recognize it as being sweet (Bazala, Knoll, \& Derndorfer, 2015). Similarly, in red wines the effect of sugar may not be recognized by consumers as a sweetener but as a smoothener. The appeal of these smoothened wines may also be inferred from the results of wine challenges, where the best awarded wines (Great Gold medal) have frequently over $10 \mathrm{~g} / \mathrm{L}$ of residual sugar (Loureiro et al., 2016). These authors also showed that average residual sugar concentrations increased in the wines awarded great gold medals from 2004 to 2014 . Therefore, preferred levels in the range determined by us under controlled conditions reflect an overall appreciation of red wines with relatively high sugar concentrations for wines considered to be dry. In fact, the definition given by OIV standards is that dry wines contain a maximum of $4 \mathrm{~g} / \mathrm{L}$ glucose plus fructose, medium dry between 4 and $12 \mathrm{~g} / \mathrm{L}$, semi-sweet between 12 and $45 \mathrm{~g} / \mathrm{L}$ and sweet when the sugar level is over $45 \mathrm{~g} / \mathrm{L}$ (OIV, 2015). Higher concentrations may apply to dry $(9 \mathrm{~g} / \mathrm{L})$ or medium dry $(18 \mathrm{~g} / \mathrm{L})$ types when the level of total acidity expressed in $\mathrm{g} / \mathrm{L}$ tartaric acid is not $<2 \mathrm{~g} / \mathrm{L}$ sugar or when the difference between the sugar and acid levels does not exceed $10 \mathrm{~g} / \mathrm{L}$, respectively. As a result, wine challenges should introduce additional categories to accommodate the supposed "dry" wines according to their true sugar content. This apparent increasing preference for sweeter wines may be due to the increase in sugar levels in the childhood diets of younger tasters, as discussed by Menella, Bobowski, and Reed (2016). Given the frequency of sweetened wines and the present dietary concerns it would be adequate to provide information about the sugar level in the wine label.

Meaningful interpretations of differences in consumer categories should have $>30$ (ISO, 1983) or 40 individuals (MacFie, 2007). Hence, our conclusions are drawn with caution for the experience category (29 experienced tasters) and the extraversion trait (26 low extraverts). The preference for sweetness appeared to be independent from consumer categories under the most common residual sugars in red wines ( $\leq 16 \mathrm{~g} / \mathrm{L}$ ), except for the trait extraversion which is expected to be linked to sweet taste preference (Saliba et al., 2009). Under the highest concentration tested $(32 \mathrm{~g} / \mathrm{L})$, wines became noticeably sweet or smooth. Females and novices showed higher preference for this sugar level when compared with males or experienced tasters, respectively. Similar results were reported by Blackman et al. (2010) regarding the experienced segment but gender was not evaluated by these authors.

Francis and Williamson (2015) considered that the inclusion of measurements of personality traits is of interest for winemakers. It is likely that people who have an interest in wine and are willing to spend quite large sums of money in buying them, will have certain psychological personality traits, such as interest in new experiences, high sociability, an intellectual interest in wine flavor differences or a desire to signal their taste to others (Francis \& Williamson, 2015). While there has been limited work investigating this area, it has been shown that there is a generally positive link between alcohol use and extraversion, impulsivity, or neuroticism and a negative link with conscientiousness (Zhang, Bray, Zhang, \& Lanza, 2015). A study involving aroma characterization and liking of a set of Italian red wines showed a link between "innovativeness" (related to openness) and consumers who could reliably differentiate samples (Torri et al., 2013). Concerning sweet taste, Saliba et al. (2009) found that participants with preference for $20 \mathrm{~g} / \mathrm{L}$ sugar in white wine reported significantly lower level of openness. In the present study individuals with low openness were insensitive to sweetness which may be explained by the different responses induced by red wines. Contrarily, high openness individuals were sweet dislikers. In another study, high scorers for openness reported healthier dietary practices since individuals with a preference for non-sweet tastes, or dry tastes, seem to be less likely to consume high-sugar products (Goldberg \& Strycker, 2002). Research by Kikuchi and Watanabe (2000) found that individuals (mostly females) scoring high in neuroticism preferred sweet tastes, and the results presented here showed that sweet likers indeed formed this segment. In addition, these results suggested some relation among openness and neuroticism. High openness and high neuroticism or low openness and low neuroticism were both associated with higher preference for sweetness, under $16 \mathrm{~g} / \mathrm{L}$ sugar. Further investigation is necessary to understand these different behaviors based on personality traits.

\section{Limitations of the study and future research}

The composition of the tasting group recruited among faculty staff, students and graduated persons may have selected individuals with higher level of education that are not representative of the overall wine consumers in Portugal. In addition, in spite of the overall high number of respondents, many categories did not reach the minimum value of 40, mainly when interactions among class categories were determined. These drawbacks should be avoided in future research by obtaining balanced number of individuals for each category. The utilization of self-reported questionnaires also tends to favour socially desirable responses but are commonly used as indicators of personality traits (Saliba et al., 2009).

For the industry and concerning fine wines, manipulation through sugar addition, although being cost-effective, is not a sophisticated way to increase smoothness and pleasantness. Hence, other strategies related with mouthfeel fine-tuning (Marchal et al., 2013; Sáenz-Navajas et al., 2012) should be tested in the future. Moreover, research is required to extend this investigation to other red wine styles, as responses are likely to be different. Ultimately, pairing with food should also be evaluated to highlight the quality of aggressive wines instead of seeking how to smooth them.

\section{Conclusions}

The results presented in this study can help winemakers to understand the importance of sugar addition in order to take into account consumers' personal preferences and hence increase wines' likeability. Residual sugar can be modulated to shape the sensory properties of wines in order to create "softer" and more desirable products. In particular, knowing that cool climate wines seem to be the next consumer trend, their acidity and astringency may be fine-tuned by sugar addition. Wine education and exposure to these wine styles, especially for new-to-wine markets, is crucial to achieve this purpose.

\section{Acknowledgments}

This work was supported by national funds from Fundação para a Ciência e a Tecnologia (FCT) through the research unit UID/AGR/ 04129/2013 (LEAF).

\section{Appendix A. Supplementary data}

Supplementary data to this article can be found online at https:// doi.org/10.1016/j.foodres.2017.12.043. 


\section{References}

Amerine, M. A., \& Ough, C. S. (1967). Sweetness preference in rosé wines. American Journal of Enology and Viticulture, 18, 121-125.

Asao, K., Miller, J., Arcori, L., Lumeng, J., Han-Markey, T., \& Herman, W. (2015). Patterns of sweet taste liking: A pilot study. Nutrients, 7(9), 7298-7311.

Bazala, B., Knoll, M., \& Derndorfer, E. (2015). Perception and acceptance of white wines by consumers belonging to different age groups. Journal International des Sciences de la Vigne et du Vin, 49, 217-230.

Blackman, J., Saliba, A., \& Schmidtke, L. (2010). Sweetness acceptance of novices, experienced consumers and winemakers in Hunter Valley Semillon wines. Food Quality and Preference, 21, 679-683.

Chung, S.-J., \& Vickers, Z. (2007). Influence of sweetness on the sensory-specific satiety and long-term acceptability of tea. Food Quality and Preference, 18, 256-264.

Costa, P. T., Jr., \& McCrae, R. R. (1992). Revised NEO personality inventory (NEO-PI-R) and NEO five-factor inventory (NEO-FFI) professional manual. Odessa, FL: Psychological Assessment Resources.

Drewnowski, A., Henderson, A., Shore, A., \& Barratt-Fornell, A. (1997). Nontasters, tasters, and supertasters of 6-n-propylthiouracil (PROP) and hedonic response to sweet. Physiology and Behaviour, 62, 649-655.

Duitschaever, C. L., Buteau, C., \& Ashton, G. C. (1980). Edulcoration of white wine with four different sweeteners. American Journal of Enology and Viticulture, 31, 337-343.

Filipello, F., Berg, H. W., Hinreiner, E., \& Webb, A. D. (1955). Reproducibility of results in consumer wine preference surveys. Food Technology, 9, 431-432.

Francis, I. L., Osidacz, P. C., Bramley, B., King, E. S., O'Brien, V., Curtin, C., ... Pretorius, I. S. (2010). Linking wine flavor components, sensory properties and consumer quality perceptions. Australian \& New Zealand Wine Industry Journal, 25, 18-23.

Francis, I. L., \& Williamson, P. O. (2015). Application of consumer sensory science in wine research. Australian Journal of Grape and Wine Research, 21, 554-567.

Freitas, V., \& Mateus, N. (2001). Structural features of procyanidin interactions with salivary proteins. Journal of Agriculture and Food Chemistry, 49, 940-945.

Goldberg, L. R., \& Strycker, L. A. (2002). Personality traits and eating habits: The assessment of food preferences in a large community sample. Personality and Individual Differences, 32, 49-65.

Harwood, M., Ziegler, G., \& Hayes, J. (2012). Rejection thresholds in chocolate milk: Evidence for segmentation. Food Quality and Preference, 26, 128-133.

Hogan, R., Johnson, J., \& Briggs, S. (Eds.). (1997). Handbook of personality psychology. California: Academic Press.

ISO (1983). Standard 5495: Sensory analysis - methodology - paired comparison test.

Jayasinghe, S., Kruger, R., Walsh, D., Cao, G., Rivers, S., Richter, M., \& Breier, B. (2017). Is sweet taste perception associated with sweet food liking and intake? Nutrients, 9(7), 750.

Kielhöfer, E. (1955). Die Restsüsse des Weines. Weinberg und Keller, 2, 358-365.

Kikuchi, Y., \& Watanabe, S. (2000). Personality and dietary habits. Journal of Epidemiology, 10, 191-198.

Kim, J. Y., Prescott, J., \& Kim, K. O. (2014). Patterns of sweet liking in sucrose solutions and beverages. Food Quality and Preference, 36, 96-103.

Lattey, K. A., Bramley, B. R., \& Francis, I. L. (2010). Consumer acceptability, sensory properties and expert quality judgements of Australian cabernet sauvignon and shiraz wines. Australian Journal of Grape and Wine Research, 16, 189-202.

Lésschaeve, I., Bowen, A., \& Bruwer, J. (2012). Determining the impact of consumer characteristics to project sensory preferences in commercial white wines. American Journal of Enology and Viticulture, 63, 487-493.

Loureiro, V., Brasil, R., \& Malfeito-Ferreira, M. (2016). A new wine tasting approach based on emotional responses to rapidly recognize classic European wine styles. Beverages, 2(1), 6.

Lund, C. M., Thompson, M. K., Benkwitz, F., Wohler, M. W., Triggs, C. M., Gardner, R., ... MacFie, H. (2007). Preference mapping and food product development. In H. Macfie (Ed.). Consumer-led food product development (pp. 551-592). Cambridge, England: Woodhead Publishing.

Marchal, A., Pons, A., Lavigne, V., \& Dubourdieu, D. (2013). Contribution of oak wood ageing to the sweet perception of dry wines. Australian Journal of Grape and Wine Research, 19, 11-19.

Macfie, H. (Ed.). (2007). Consumer-led food product development. Cambridge, England: Woodhead Publishing.

Melcher, J. M., \& Schooler, J. W. (1996). The misremembrance of wines past: Verbal and perceptual expertise differentially mediate verbal overshadowing of taste memory. Journal of Memory and Language, 35, 231-245.

Menella, J., Bobowski, N., \& Reed, D. (2016). The development of sweet taste: From biology to hedonics. Reviews in Endocrinology and Metabolism Disorder, 17, 171-178.

Methven, L., Xiao, C., Cai, M., \& Prescott, J. (2016). Rejection thresholds (RjT) of sweet likers and dislikers. Food Quality and Preference, 52, 74-80.

Moskovitz, H. R., Jacobs, B. E., \& Lazar, N. (1985). Product response segmentation and the analysis of individual differences in liking. Journal of Food Quality, 8, 191-198.

OIV (2010). Recueil des méthodes internationales d'analyse des vins et des moûts. International Organisation of Vine and Wine, Paris, France.

OIV (2015). International standard for the labelling of wines. Paris, France: International Organisation of Vine and Wine.

Osidacz, P., Francis, I. L., Bramley, B., \& Stevens, M. (2011). The effect of repeated wine exposure on consumer preferences. In R. J. Blair, T. H. Lee, \& I. S. Pretorius (Eds.). Proceedings of the fourtheenth Australian wine industry technical conference; 3-8 July 2010; Adelaide, SA, Australia (Australian Wine Industry Technical Conference: Urrbrae, SA, Australia) (pp. 223-225). .

Prescott, J. (1998). Comparisons of taste perceptions and preferences of Japanese and Australian consumers: Overview and implications for cross-cultural sensory research. Food Quality and Preference, 9, 393-402.

Prescott, J., Laing, D., Bell, G., Yoshida, M., Gillmore, R., \& Allen, S. (1992). Hedonic responses to taste solutions: A cross-cultural study of Japanese and Australian panels. Chemical Senses, 17, 801-809.

Prescott, J., Norris, L., Kunst, M., \& Kim, S. (2005). Estimating a "consumer rejection threshold" for a cork taint in white wine. Food Quality and Preference, 16, 345-349.

Ribéreau-Gayon, P. (1970). La dosage des composés phenoliques totaux das les vins rouges. Chemie Analytique, 52, 627-631.

Sáenz-Navajas, M. P., Fernandez-Zurbano, P., \& Ferreira, V. (2012). Contribution of nonvolatile composition to wine flavor. Food Reviews International, 28, 389-411.

Saliba, A., Wragg, K., \& Richardson, P. (2009). Sweet taste preference and personality traits using a white wine. Food Quality and Preference, 20, 572-575.

Somers, T. C., \& Evans, M. E. (1977). Spectral evaluation of young red wines: Anthocyanin equilibria, total phenolic, free and molecular SO2, "chemical age". Journal of the Science of Food and Agriculture, 28, 279-287.

Thompson, D. A., Moskovitz, H. R., \& Campbell, R. (1976). Effects of body weight and food intake on pleasantness ratings for a sweet stimulus. Journal of Applied Psychology, 41, 77-83.

Torri, L., Dinnella, C., Recchia, A., Naes, T., Tuorila, H., \& Monteleone, E. (2013). Projective mapping for interpreting wine aroma differences as perceived by naïve and experienced assessors. Food Quality and Preference, 29, 6-15.

Wansink, B., Bascoul, G., \& Che, G. (2006). The sweet tooth hypothesis: How fruit consumption relates to snack consumption. Appetite, 47, 107-110.

Whiterly, S., Pangborn, R., \& Stern, J. (1980). Gustatory responses and eating duration of obese and lean adults. Appetite, 1, 53-63.

Williamson, P. O., Robichaud, J. A., \& Francis, I. L. (2012). Comparison of Chinese and Australian consumer's liking responses for red wines. Australian Journal of Grape and Wine Research, 18, 256-267.

Yeomans, M., Prescott, J., \& Gould, N. (2009). Acquired hedonic and sensory characteristics of odours; influence of sweet liker and propylthiouracil taster status. The Quartely Journal of Experimental Psychology, 62, 1648-1664.

Yeomans, M., Tepper, B., Riestzschel, J., \& Prescott, J. (2007). Human hedonic respenses to sweetness: Role of taste genetics and anatomy. Physiology and Behaviour, 91, 264-273.

Zhang, J., Bray, B. C., Zhang, M., \& Lanza, S. T. (2015). Personality profiles and frequent heavy drinking in young adulthood. Personality and Individual Differences, 80, 18-21. 